\title{
Fatores que provocam resultados falso-negativos nos exames de citologia oncótica: uma revisão integrativa
}

\author{
Factors that cause false-negative results in oncotic cytology exams: an integrative review \\ Factores que causan resultados falso-negativos en exámenes de citología oncótica: una revisión
}

integrativa

Recebido: 04/07/2021 | Revisado: 08/08/2021 | Aceito: 09/08/2021 | Publicado: 14/08/2021

Maria Cristiane Oliveira Costa

ORCID: https://orcid.org/0000-0003-3154-2315 Faculdade Laboro, Brasil

E-mail: Mariacristianebiomed@hotmail.com

Carla Mayla Silva de Melo

ORCID: https://orcid.org/0000-0002-3421-669X Faculdade Laboro, Brasil

E-mail: carlamayla17@hotmail.com

Eduarda dos Santos Lima

ORCID: https://orcid.org/0000-0003-1252-9959 Universidade Federal do Maranhão, Brasil

E-mail: eduardalimabiomedicina97@gmail.com

Jean Carlos Rodrigues da Cunha

ORCID: https://orcid.org/0000-0002-0741-3583 Faculdade Futura, Brasil

E-mail: je.carl@outlook.com.br

Ana Paula Muniz Serejo

ORCID: https://orcid.org/0000-0002-4376-4364 Universidade Federal do Maranhão, Brasil E-mail: apsmuniz1@gmail.com

Heliana de Araújo Morais

ORCID: https://orcid.org/0000-0001-6158-1804 Universidade Federal do Maranhão, Brasil

E-mail: helianamorais@gmail.com

\begin{abstract}
Resumo
O câncer do colo do útero (CCU) é considerado um grave problema de saúde pública no mundo sendo a terceira malignidade de maior índice de mortalidades entre as mulheres brasileiras. O exame citopatológico, mas conhecido como exame do Papanicolaou, é uma estratégia valiosa para o diagnóstico precoce do $\mathrm{CCU}$, uma vez que se trata de um método com alta precisão e baixo custo. A pesquisa tem como objetivo revisar a literatura quanto os possíveis fatores que podem levar a resultados falso-negativos no exame do Papanicolaou. Foi realizada revisão integrativa nas bases de dados on-line: Literatura Latino-Americana e do Caribe em Ciências da Saúde (LILACS); Medical Literature Analysis and Retrieval System Online (MEDILINE) e Scientific Electronic Library Online (SciELO). Sendo selecionadas 15 artigos com publicação entre os anos de 2015 a 2020, nos idiomas inglês e português. Os principais fatores responsáveis por ocasionar resultados falso - negativos referem-se a erros ocorridos nas fases pré-analítica, analítica e pós-analítica, a execução da coleta com uma amostra inadequada, é uma fase relevante que impacta na análise do esfregaço quando o profissional interpreta erroneamente os exames, geralmente em decorrência de uma coloração inadequada e uma confecção imprópria das lâminas ou ainda por prática e formação insuficiente do citologista. A qualidade do exame Papanicolaou depende do controle de qualidade dos exames citopatológicos, pois este se baseia em medidas para detectar, corrigir e reduzir erros ocasionados nas fases: pré-analítica; analítica e pósanalítica evitando, assim, os resultados falsos-negativos. Além disso, a capacitação contínua dos profissionais atuantes na área da saúde reflete diretamente na boa qualidade e confiabilidade dos exames em geral, tendo ênfase aqui, para o exame Papanicolaou.
\end{abstract}

Palavras-chave: Teste de Papanicolaou; Neoplasias do colo do útero; Controle de qualidade, Controle analítico de qualidade.

\footnotetext{
Abstract

Cervical cancer (CCU) is considered a serious public health problem in the world, being the third malignancy with the highest mortality rate among Brazilian women. The Pap smear test, known as the Papanicolaou test, is a valuable strategy for the early diagnosis of CC, since it is a method with high precision and low cost. The research aims to review the literature regarding possible factors that can lead to false-negative results in the Pap smear. An integrative
} 
review was carried out in the online databases: Latin American and Caribbean Literature on Health Sciences (LILACS); Medical Literature Analysis and Retrieval System Online (MEDILINE) and Scientific Electronic Library Online (SciELO). 15 articles were selected and published between the years 2015 to 2020, in English and Portuguese. The main factors responsible for false results - negative refers to errors that occurred in the pre-analytical, analytical and post-analytical phases, the execution of the collection with an inadequate sample, is a phase that impacts the analysis of the smear when the professional misinterprets the tests, usually due to an inadequate color and improper preparation of the slides or even by practice and insufficient training. The quality of the Pap smear depends on the quality control of cytopathological tests, as this is based on measures to detect, correct and reduce errors caused in the phases: pre-analytical; analytical and post-analytical, thus avoiding false-negative results.In addition, the continuous training of professionals working in the health area directly reflects on the good quality and reliability of exams in general, with emphasis here on the Pap smear.

Keywords: Pap smear test; Cervical neoplasms; Quality control; Analytical quality control.

\section{Resumen}

El cáncer de cuello uterino (UCC) es considerado un grave problema de salud pública en el mundo, siendo la tercera neoplasia con mayor tasa de mortalidad entre las mujeres brasileñas. La prueba de Papanicolaou, conocida como prueba de Papanicolaou, es una estrategia valiosa para el diagnóstico precoz de CC, ya que es un método de alta precisión y bajo costo. La investigación tiene como objetivo revisar la literatura sobre posibles factores que pueden conducir a resultados falsos negativos en la prueba de Papanicolaou. Se realizó una revisión integradora en las bases de datos en línea: Literatura Latinoamericana y del Caribe en Ciencias de la Salud (LILACS); Sistema de Análisis y Recuperación de Literatura Médica en Línea (MEDILINE) y Biblioteca Electrónica Científica en Línea (SciELO). Se seleccionaron y publicaron 15 artículos entre los años 2015 a 2020, en inglés y portugués. Los principales factores responsables de los resultados falsos - negativo se refiere a los errores que se produjeron en las fases preanalítica, analítica y postanalítica, la ejecución de la colección con una muestra inadecuada, es una fase que impacta en el análisis del frotis cuando el profesional malinterpreta las pruebas, generalmente debido a un color inadecuado y una preparación inadecuada de los portaobjetos o incluso por la práctica y la capacitación insuficiente. La calidad de la prueba de Papanicolaou depende del control de calidad de las pruebas citopatológicas, ya que este se basa en medidas para detectar, corregir y reducir los errores causados en las fases: preanalíticas; analíticos y post-analíticos, evitando así resultados falsos negativos. Además, la formación continua de los profesionales que trabajan en el área de la salud se refleja directamente en la buena calidad y confiabilidad de los exámenes en general, con énfasis aquí en la prueba de Papanicolaou.

Palabras clave: Prueba de Papanicolaou; Neoplasias cervicales; Control de calidad; Control de calidad analítico.

\section{Introdução}

O câncer de colo uterino (CCU) é considerado um grave problema de saúde pública no mundo, principalmente em países subdesenvolvidos, devido às altas taxas de mortalidade, à exposição de mulheres a fatores de riscos como infecção pelo vírus do papiloma humano (HPV), histórico sexual, tabagismo, imunossupressão, infecção por clamídia, fármacos anticoncepcionais, múltiplas gestações, idade, fatores socioeconômicos e má alimentação são indicadores que contribuem para alta incidência de neoplasia do colo do útero (Amaral, et al. 2014).

Mundialmente, o CCU é o segundo tipo de neoplasia com os maiores índices de ocorrência concentrados na América Latina, Caribe, África e no Sul e Sudeste da Ásia de acordo com dado da International Agency for Cancer Research (IARC) no ano de 2019. Em um estudo realizado por Magalhães (2020), os pesquisadores apontam essa neoplasia é a terceiro tumor maligno mais frequente na população feminina em território brasileiro e segundo as estimativas da IARC, a ocorrência seria de 17.030 novos casos de CCU, no ano de 2020 (International Agency for Cancer Research - IARC, 2019; Magalhães, 2020).

O exame citopatológico popularmente conhecido como "preventivo" é o método mais usado para o rastreamento de malignidades do colo uterino e de lesões precursoras, método introduzido desde o ano de 1941, desenvolvido por Papanicolaou - médico grego que iniciou o rastreio do carcinoma do colo do útero, em 1927, após observar alterações nas células das regiões da cérvix e vagina e em diferentes fases do ciclo menstrual. Os seus procedimentos de coleta, preparo, leitura e liberação do exame são totalmente manuais, tornando-o mais propenso a erros. Por esse motivo, todas as fases do exame laboratorial devem ser corretamente realizadas, seguindo todos os requisitos do controle de qualidade dos exames citopatológicos, para minimizar os resultados falsos-negativos e assim garantir a eficiência no rastreamento do CCU (Silva; Assis, 2019). 
Haja vista a importância do exame, a qualidade da amostra é um ponto crucial para o desfecho do rastreamento do $\mathrm{CCU}$, uma vez que a presença das células escamosas, glandulares e/ou metaplásicas, bem distribuídas, fixadas, coradas de forma adequada e com quantidade representativa das células é de fundamental importância, para identificação das lesões percursoras de carcinomas cervicais localizados na zona de transformação, mesmo em casos assintomáticos, diminuindo assim, a incidência e mortalidade por esse câncer (Ughini, 2016; Santos e Ribeiro, 2020).

Quando o CCU é identificado de forma precoce, considera-se como um melhor prognóstico, sendo necessário que o exame citopatológico seja realizado seguindo os preceitos da Gestão da Qualidade e com a periodicidade recomendada pelo Ministério da Saúde. Variáveis nas fases pré-analítica e analítica como erro de coleta, fixação, montagem da lâmina, pouca experiência do profissional, podem comprometer a análise obtendo um resultado falso-negativo (Jakobczynski, 2018; Magalhães, 2020).

A baixa sensibilidade do exame de Papanicolaou tem sido alvo de várias críticas, pois as suas taxas podem variar entre $6 \%$ a $56 \%$ de resultados falsos-negativos ocorrendo tanto na fase pré-analítica como na analítica. Pode-se citar como alguns dos erros mais comum na fase pré-analítica o tempo prolongado entre a coleta e a fixação, erros no modo de uso do fixador ou ainda manuseio incorreto e falta da manutenção adequada dos corantes. Já na fase analítica pode-se apontar como a principal falha a interpretação de células neoplásicas como benignas (SILVA e Assis, 2019; Machado et al. 2018).

A Organização Mundial de Saúde (OMS) preconiza a execução do exame citopatológico de maneira correta, atingindo $80 \%$ de cobertura e executado dentro parâmetros de qualidade. No Brasil, o Ministério da Saúde através da Portaria MS nº 3.388 de 30 de dezembro de 2013, formulou a Qualificação nacional em citopatologia na prevenção do câncer do colo do útero - Qualicito (Santos; Ribeiro, 2020).

Considerando o exposto, mesmo com os esforços e recomendações da OMS e legislação brasileira vigente para melhorias nos exames citopatológicos, ainda as causas que elevam a incidências dos resultados errôneos persistem como um desafio enfrentado nos laboratórios e pelas equipes de saúde do país. Logo se faz necessário estudos para traçar estratégias e reduzir os resultados falso-negativos no exame Papanicolaou. O objetivo deste estudo é identificar os fatores existentes na literatura que provocam resultados falso-negativos no exame de citologia oncótica.

\section{Metodologia}

A revisão integrativa da literatura foi realizada no segundo semestre de 2020, através de consultas nos seguintes portais: Portal Regional da Biblioteca Virtual em Saúde (BVS), Centro Latino Americano de Informações em Ciências da Saúde (BIREME), Organização Pan-Americana de Saúde (OPAS)/ Organização Mundial de Saúde (OMS). Para pesquisas das obras, utilizaram-se as bases de dados on-line: Literatura Latino-Americana e do Caribe em Ciências da Saúde (LILACS); Medical Literature Analysis and Retrieval System Online (MEDILINE), Scientific Electronic Library Online (SciELO) e Google Acadêmico.

As buscas dos artigos foram realizadas aplicando as palavras-chaves presentes nos Descritores em Ciências da Saúde (DeCS / MeSH), versão 2020: Teste de Papanicolaou; Neoplasias do Colo do Útero; Controle de Qualidade, Controle Analítico de Qualidade. Os descritores foram usados nos idiomas inglês e português em todas as bases de dados, comodemostra o quadro abaixo, empregando como exemplo a base LILACS. 
Quadro 1. Demonstração da busca utilizando os descritores na base de dados LILACS.

\begin{tabular}{|c|c|c|}
\hline $\begin{array}{c}\text { IDIOMA / } \\
\text { BASE DE DADOS }\end{array}$ & DESCRITORES & $\begin{array}{l}N^{0} \text { DE ARTIGOS } \\
\text { ENCONTRADOS }\end{array}$ \\
\hline \multirow{6}{*}{$\begin{array}{l}\text { Português / } \\
\text { LILACS }\end{array}$} & Teste $A N D$ de $A N D$ Papanicolaou. & 241 \\
\hline & $\begin{array}{l}\text { Neoplasias } A N D \text { do } A N D \text { Colo } A N D \text { do } A N D \text { Útero } O R \\
\text { Câncer } A N D \text { de } A N D \text { Colo } A N D \text { Uterino. }\end{array}$ & \\
\hline & Fase $A N D$ Pré-Analítica $O R$ Fase $A N D$ pós- Analítica & 38 \\
\hline & $\begin{array}{l}\text { Fase } A N D \text { Pré-Analítica } O R \text { Fase } A N D \text { pós- Analítica } A N D \\
\text { Teste } A N D \text { de } A N D \text { Papanicolaou. }\end{array}$ & 1 \\
\hline & $\begin{array}{l}\text { Controle } A N D \text { de } A N D \text { Qualidade } O R \text { Controle } A N D \\
\text { Analítico } A N D \text { de } A N D \text { Qualidade. }\end{array}$ & \\
\hline & $\begin{array}{l}\text { Controle } A N D \text { de } A N D \text { Qualidade } O R \text { Controle } A N D \\
\text { Analítico } A N D \text { de } A N D \text { Qualidade } A N D \text { Teste } A N D \text { de } A N D \\
\text { Papanicolaou. }\end{array}$ & 10 \\
\hline \multirow{6}{*}{$\begin{array}{l}\text { Inglês / } \\
\text { LILACS }\end{array}$} & test $A N D$ of $A N D$ Papanicolaou & 0 \\
\hline & $\begin{array}{l}\text { Neoplasms } A N D \text { of } A N D \text { Colo } A N D \text { of } A N D \text { Uterus } O R \\
\text { Cancer } A N D \text { of } A N D \text { Colo } A N D \text { Uterine. }\end{array}$ & 3.398 \\
\hline & $\begin{array}{l}\text { Pre-Analytical } A N D \text { Phase } O R \text { Post-Analytical } A N D \text { Phase } \\
A N D \text { Test } A N D \text { Papanicolaou. }\end{array}$ & \\
\hline & $\begin{array}{l}\text { Quality } A N D \text { Control } O R \text { Analytical } A N D \text { Quality } A N D \\
\text { Control. }\end{array}$ & \\
\hline & $\begin{array}{l}\text { Quality } A N D \text { Control } O R \text { Analytical } A N D \text { Quality } A N D \\
\text { Control } A N D \text { Test } A N D \text { Papanicolaou. }\end{array}$ & 4 \\
\hline & Total: & 22.918 \\
\hline
\end{tabular}

Fonte: Autores (2021).

As obras selecionadas foram submetidas aos critérios de inclusão e exclusão. Para tal, foram considerados os seguintes critérios de inclusão: a) artigos disponíveis na literatura relacionados a qualidade dos exames de citologia oncóticas, bem como os fatores que contribuem para os resultados falsos-negativos; b) estudos com publicação entre os anos de 2015 a 2020 e c) publicações nos idiomas inglês e português. Por outro lado, teses, dissertações, resumos de anais e estudos que não contemplavam o tema de forma suscita, de acordo com os critérios de inclusão, foram excluídos. Assim, após análise foram selecionados um total de 15 artigos por atenderem os critérios e subsidiaram a escrita do trabalho.

As obras originais selecionadas foram analisadas através de uma leitura inicial, dos títulos e resumos para uma préseleção dos artigos. Em uma segunda leitura foi realizado a análise dos dados disponíveis nos resultados para um melhor entendimento do método utilizado nos estudos. Posteriormente, realizou-se a categorização pela relevância do assunto das obras, a fim de atender a temática do presente estudo.

\section{Resultados e Discussão}

Seguindo tais premissas, ao realizar a leitura dos 15 artigos, foi observado que o exame de Papanicolaou é de grande relevância na detecção precoce de lesões que podem indicar uma neoplasia uterina e que as principais causas dos resultados 
falsos-negativos estão relacionadas a erros ocorridos no ato da coleta, no escrutínio (Leitura da lâmina de citologia de um lado para outro ou de cima para baixo. O profissional deve examinar a lâmina, progredindo campo a campo, até que toda a área sob a lamínula seja inspecionada), do esfregaço e na interpretação dos exames.

Nota-se a importância das fases pré-analítica, analítica e pós-analítica no exame de Papanicolaou, uma vez que para atingir a meta satisfatória da investigação é fundamental avaliar todas as etapas nas quais pode ocorrer falhas. Dessa forma, optou-se por categorizar as obras analisadas e consideradas neste estudo, com intuito de usufruir melhor das informações, como está exporto na Tabela 1.1.

Tabela 1. Distribuição das obras selecionadas com principais fatores apontados através dos artigos selecionados.

\begin{tabular}{|c|c|c|}
\hline & AUTORES & TÍTULOS \\
\hline 1 & $\begin{array}{l}\text { Branca e } \\
\text { Longatto-Filho, } \\
2015 .\end{array}$ & $\begin{array}{c}\text { Recommendations on Quality Control } \\
\text { and Quality Assurance in Cervical } \\
\text { Cytology. }\end{array}$ \\
\hline 2 & $\begin{array}{l}\text { Clarke et al. } \\
2018 .\end{array}$ & $\begin{array}{c}\text { Epidemiologic Evidence That Excess } \\
\text { Body Weight Increases Risk of } \\
\text { Cervical Cancer by Decreased } \\
\text { Detection of Precancer. }\end{array}$ \\
\hline 3 & $\begin{array}{c}\text { Galvão et AL, } \\
2015 .\end{array}$ & $\begin{array}{c}\text { Frequência de amostras insatisfatórias } \\
\text { dos exames preventivos do câncer de } \\
\text { colo uterino na rede pública de saúde, } \\
\text { em município do agreste } \\
\text { pernambucano }\end{array}$ \\
\hline 4 & $\begin{array}{c}\text { Jakobczynski, } \\
2018 .\end{array}$ & $\begin{array}{l}\text { Capacitação dos profissionais de } \\
\text { saúde e seu impacto no rastreamento } \\
\text { de lesões precursoras do câncer de } \\
\text { colo uterino. }\end{array}$ \\
\hline 5 & $\begin{array}{c}\text { Machado et al, } \\
2018\end{array}$ & $\begin{array}{l}\text { Controle interno da qualidade em } \\
\text { citopatologia: o dilema da } \\
\text { subjetividade. }\end{array}$ \\
\hline 6 & $\begin{array}{l}\text { Magalhães, } \\
2020 .\end{array}$ & $\begin{array}{l}\text { Avaliação dos indicadores de } \\
\text { qualidade dos exames citopatológicos } \\
\text { do colo do útero realizados em um } \\
\text { município do Paraná, Brasil }\end{array}$ \\
\hline
\end{tabular}

\begin{tabular}{|c|c|c|}
\hline 7 & $\begin{array}{l}\text { Mori e Ribeiro, } \\
2015 .\end{array}$ & $\begin{array}{l}\text { Falhas no diagnóstico do câncer de } \\
\text { colo uterino. }\end{array}$ \\
\hline 8 & $\begin{array}{l}\text { Paula, et al, } \\
\quad 2017 .\end{array}$ & $\begin{array}{l}\text { Indicadores do monitoramento } \\
\text { interno da qualidade dos exames } \\
\text { citopatológicos do Laboratório } \\
\text { Clínico da Pontifícia Universidade } \\
\text { Católica de Goias (PUC-GO). }\end{array}$ \\
\hline 9 & Roever, 2017. & $\begin{array}{l}\text { Compreendendo os estudos de revisão } \\
\text { sistemática }\end{array}$ \\
\hline 10 & $\begin{array}{l}\text { Rocha et al, } \\
\quad 2018 .\end{array}$ & $\begin{array}{l}\text { External Quality Monitoring of the } \\
\text { Cervical Cytopathological Exams in } \\
\text { the Rio de Janeiro City. }\end{array}$ \\
\hline 11 & Silva E Assis & $\begin{array}{l}\text { Os efeitos das incorreções pré- } \\
\text { analíticas para o exame de } \\
\text { papanicolaou. }\end{array}$ \\
\hline
\end{tabular}

RESUMO DAS OBRAS
O Controle de qualidade representa uma ferramenta
fundamento para minimizar os erros falsos-negativos
ou falsos positivos nos laboratórios de citopatologia.
O teste do papanicolau realizado corretamente e livres
de erros é fundamento para o diagnóstico precoce do
câncer do colo do útero, principalmente em pacientes
obesas, pois possuem maior predisposição para
desenvolver a doença.

A qualidade da amostra está relacionada a capacitação do profissional de citopatologia, sendo essa primeira etapa primordial para uma boa excussão do exame e consequentemente amenizar os resultados falsosnegativos.

Observa-se que a capacitação dos profissionais de citologia melhora a adequabilidade da amostra, representação dos epitélios e resultado dos exames com a consequente diminuição dos resultados falsosnegativos.

O estudo ressalta para a importância de um sistema de controle de qualidade em citopatologia, com a finalidade de amenizar os laudos falso-negativos.

Os resultados do estudo apontam que nos anos houve um aumento na detecção das lesões intraepiteliais escamosas de alto grau (HSIL) e das células escamosas atípicas (ASC) com características suspeitas de invasão, implicando diretamente na diminuição dos resulta falsos-negativos.

A etapa da coleta é responsável por $20 \%$ a $39 \%$ dos resultados falso-negativos, sendo talvez, a mais importante, pois ela precede todos os outros passos. O monitoramento interno da qualidade é uma ferramenta usada no auxílio diagnóstico, garantindo assim uma maior acurácia dos exames citopatológicos diminuído, assim, os resultados falsos-negativos.

A revisão sistemática é um método rigoroso de resumir as evidências cientificas presente na literature, utilizando metodologia clara e pré-definida.

$\mathrm{O}$ estudo demostro que o monitoramento externo de qualidade foi fundamental para garantia e a confiabilidade dos exames citopatológicos nos laboratórios avaliados na cidade do Rio de Janeiro.

A não detecção de células anormais nos diagnósticos podem adiar o tratamento sendo prejudicial as pacientes. Assim, é necessário que medidas de controle 


\begin{tabular}{|c|c|c|c|}
\hline & & & $\begin{array}{l}\text { de qualidade sejam implementadas, visando reduzir a } \\
\text { liberação de falsos resultados. }\end{array}$ \\
\hline 12 & $\begin{array}{l}\text { Silva, } \\
\text { Cristovam E } \\
\text { Vidotti, } 2017 .\end{array}$ & $\begin{array}{c}\text { O impacto da fase pré-analítica na } \\
\text { qualidade dos esfregaços } \\
\text { cervicovaginais }\end{array}$ & $\begin{array}{l}\text { É fundamental, que o desenvolvimento de uma rotina } \\
\text { de controle de qualidade em todo o processo do exames } \\
\text { papanicolau, incluindo a fase pré-analítica, visando } \\
\text { uma maior segurança nos resultados dos exames. }\end{array}$ \\
\hline 13 & $\begin{array}{c}\text { Santos e } \\
\text { Ribeiro, } 2020 .\end{array}$ & $\begin{array}{c}\text { Estratégias Utilizadas para Melhorar a } \\
\text { Qualidade dos Exames } \\
\text { Citopatológicos. }\end{array}$ & $\begin{array}{l}\text { É de suma importância a capacitação continuada dos } \\
\text { profissionais envolvidos todas as etapas do processo, da } \\
\text { fase pré-analítica à analítica, com a finalidade de } \\
\text { reduzir os erros dos exames citopatológicos. }\end{array}$ \\
\hline 14 & Ughini, 2016. & $\begin{array}{l}\text { Importância da qualidade da coleta do } \\
\text { exame preventivo para o diagnóstico } \\
\text { das neoplasias glandulares } \\
\text { endocervicais e endometriais. }\end{array}$ & $\begin{array}{l}\text { Observou-se a necessidade de melhor orientação e } \\
\text { capacitação dos profissionais responsável pela coleta, } \\
\text { uma vez que erro ocasionados nessa fase podem } \\
\text { desencadear possíveis resultados falsos-negativos. }\end{array}$ \\
\hline 15 & Wilson, 2015. & $\begin{array}{l}\text { The role of Cytotechnologists in } \\
\text { quality assurance and audit in non- } \\
\text { gynaecological cytology. }\end{array}$ & $\begin{array}{l}\text { Os padrões de qualidade devem ser prioridade } \\
\text { contínuos. Além disso, o treinamento de pessoal é de } \\
\text { suma importância para manter a qualidade dos exames. }\end{array}$ \\
\hline
\end{tabular}

Fonte: Autores (2021).

\subsection{Erros nas fases pré-analítica, analítica e pós-analítica encontrados na literatura analisada.}

A fase pré-analítica refere-se ao conjunto de métodos que visam a qualidade e representatividade da amostra, cabendo ao profissional da saúde, responsável por executar o exame, a utilização e domínio dos critérios impostos pelo Controle Interno da Qualidade referente ao registro do material recebido, a fixação, coloração e a confecção das lâminas (Silva; Cristovam e Vidotti, 2017).

Dessa forma, o Ministério da Saúde recomenda algumas medidas relevantes que os profissionais devem adotar na fase pré-analítica, como o repasse de instruções ao público feminino sobre os cuidado prévios para realização do exame citopatológico tais quais: não utilizar medicamentos via vaginal, não realizar relação sexual nos últimos dois dias que antecedem o exame, não realizar o exame em período menstrual e atenção quanto a sangramentos anormais, nesse caso pode ser feito o exame com intuito de diagnosticar a causa do sangramento (Silva e Assis, 2019; Ministério Da Saúde, 2012).

Levando em consideração a importância da coleta, o Ministério da Saúde recomenda que o material coletado precisa ser espalhado sobre a lâmina uniformemente, fixado imediatamente após ser coletado e levado ao laboratório em suporte de lâmina com a identificação e as informações do paciente. Por outro lado, os fatores que geralmente representam erros na coleta, acarretando equívocos no escrutínio e diagnóstico, estão relacionados com a presença de sangue, ausência das células endocervicais e da zona de transformação e presença de artefatos de fixação e processos inflamatórios (Santos e Ribeiro, 2020).

Posterior a coleta, inicia-se a etapa de fixação, essencial e de suma importância no resultado final do exame, uma vez que o fixador reage com inúmeros componentes celulares promovendo a estabilidade molecular e prevenindo a degradação e morte das células. De acordo com Silva, Cristovam e Vidotti (2017), existem três categorias de fixação: fixação úmida, fixação úmida com secagem ao ar e fixação por nebulização ou cobertura do esfregaço (Mori e Ribeiro, 2015; Silva, Cristovam e Vidotti, 2017).

O procedimento de coloração finaliza a metodologia do exame de Papanicolaou, sendo desenvolvida em etapa de hidratação, coloração e desidratação das amostras com a finalidade de promover uma melhor evidência da morfologia e metabolismo das células. Para o Instituto Nacional Do Câncer - Inca a não identificação de células anormais ou neoplásicas no procedimento de escrutínio de rotina decorrente de má coloração da lâmina é um erro de alto potencial para resultado falsonegativo. Por esse motivo, cada etapa da coloração deve ser executada seguindo rigorosamente o controle de qualidade dos 
corantes utilizados na bateria de coloração, além de ser avaliado diariamente em microscópio (Silva, Cristovam e Vidotti, 2017; Instituto Nacional do Câncer - INCA, 2016).

Quanto às fases analíticas e pós-analíticas, estudos recentes frisam o papel do profissional citologista para uma boa leitura das lâminas e consequentemente resultado adequado do exame, uma vez que erros no ato do escrutínio estão relacionados a falta de atenção, prática e reconhecimento de células anormais presentes na amostra (Santos e Ribeiro, 2020).

Para Machado et al. (2018), erros relacionados à interpretação ocorrem devido à falta de experiência e déficit de informação clínica do escrutinado, fatores observados quando o profissional classifica células neoplásicas em benignas desencadeando resultados falso-negativos. Dessa forma, a capacitação do citologista é fundamental para atenuar as dificuldades enfrentadas pelo profissional e melhorar qualidade do exame citológico e dos demais procedimentos realizados em laboratório, promovendo assim, uma maior aptidão e segurança na identificação de células atípicas ao analisar as amostras.

De certo, a técnica do exame citológico possui alto grau de complexidade, sendo exigido, tanto do profissional que irá coletar e preparar a o material quanto o que irá realizar a leitura, conhecimento sobre as partes anatômicas, características da amostra e o reconhecimento da fisiologia celular presente na região cervical. Para Souza et al (2008) apud Jakobczynski et al. (2018), a formação continuada é essencial aos profissionais da saúde atuantes na prevenção do câncer do colo uterino, ressaltando ainda, que somente assim o citologista estará seguro quanto a execução do exame e a utilização adequada dos materiais necessários (Jakobczynski et al. 2018).

A Tabela 2 demonstra os erros mais comuns identificados na literatura cientifica quanto aos procedimentos das fases pré-analítica e analítica que podem desencadear resultados falsos-negativos no exame Papanicolaou.

Tabela 2. Distribuição dos estudos por fase e tipo de erro identificado.

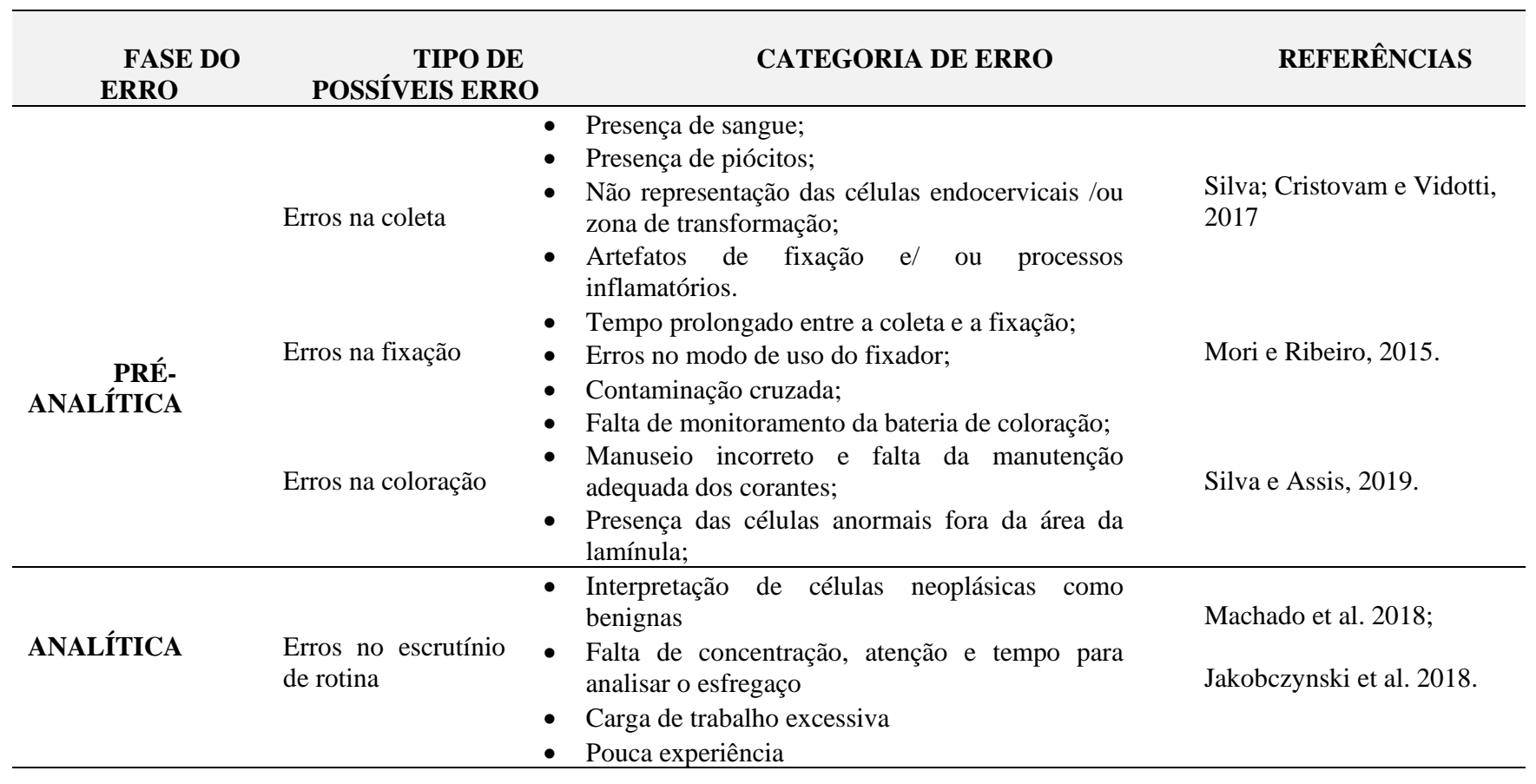

Fonte: Autores (2021).

Em um estudo realizado por Galvão e colaboradores (2015), analisando as amostras insatisfatórias dos exames citopatológico cervical, identificou no período de ano um total de 381 amostras consideradas insatisfatórias. A análise das amostras insatisfatórias demostra que 57 tiveram a leitura prejudicada (mais de $75 \%$ do esfregaço) por presença de artefatos de dessecamento, piócitos e sangue; 127 foram consideradas insatisfatórias devido a material acelular ou hipercelular; 81 foram 
rejeitadas por ausência ou erro na identificação da lâmina, frasco ou formulário. Assim, os estudos analisados apontam a efetividade dos erros majoritariamente na fase pré-analítica, sendo assim, de extrema importância a atenção, exatidão e competência dos profissionais para evitar os resultados falsos-negativos.

Para Ughini (2016), a presença de sangue ou piócitos estão relacionados a erros na coleta e são fatores prejudiciais na identificação de lesões citológicas e células anormais acarretando resultados falso-negativos, podendo ocorrer devido a pouca experiência do profissional. Outro fator importante relacionado a coleta é a ausência de células endocervicais demostrando a importância da capacitação continuado dos profissionais da área.

\subsection{Controle de qualidade e alternativas para amenizar erros no exame papanicolaou}

Do ponto de vista do Controle de Qualidade na citologia brasileira, este refere-se a correção e redução de erros na métodologia dos exames citologicos, possibilitando mais confiabilidade aos procedimentos execultados. A introdução do Controle de Qualidade no Brasil ocorreu em 2001 através da Portaria SPS/SAS nº 92 do Ministério da Saúde (MS), cuja retificação em 2013 instituiu a Qualificação nacional em citopatologia na prevenção do câncer do colo do útero (Qualicito) com a Portaria MS nº 3.388 de 30 de dezembro de 2013. O Qualicito define os padrões de avaliação da qualidade dos exames citopatológicos nos laboratórios públicos e privados que oferecem serviços ao Sistema Único de Saúde - SUS (Ministério da Saúde, 2013).

Com o intuito de orientar os laboratórios vinculados os SUS a respeito do Monitoramento Interno da Qualidade (MIQ), o Inca em parceria com o Ministério da Saúde desenvolveu o Manual de Gestão de Qualidade para Laboratórios de Citopatologia, a fim de reduzir erros, principalmente nas fases pré-analítica e analítica do exame citológico (Magalhães et al, 2020).

Além disso, de acordo com Agência Nacional de Vigilância Sanitária - ANVISA (RDC 302/ 2005), os programas de controle interno e externo de qualidade devem ser implantados e executados nos laboratórios clínicos, com intuito de identificar e eliminar os erros que possam ocorrer em todas as etapas do processo dos exames, tendo ênfase aqui para o exame Papanicolaou (ANVISA, 2005).

Todavia, apesar dos esforços para melhorar os exames e diagnósticos em Citopatologia, pesquisas realizadas no Brasil entre os anos de 2012 a 2015 demostraram índice de Positividade - IP em níveis baixos, demostrando uma não consolidação do controle interno de qualidade até o dado período. Em um estudo realizado por Bortolon at al. (2012) apud Paula et al. (2017), relata que 53\% dos laboratórios brasileiros apresentavam IP abaixo de 2\%, sendo na região centro-oeste os laboratórios possuíam uma positividade pouco maior que 3\%. Um IP baixo indica que alteração malignas não estavam sendo monitoradas, sendo portando ocasião de números alarmantes dos resultados falsos-negativos (Paula et al. 2017).

Tendo em vista a importância da citopatologia na previsão e diagnóstico precoce do CCU, muitos pesquisadores passaram a dedicar tempo na busca de melhorias dos indicadores de qualidade nos laboratórios. Nesse sentido, novas pesquisas apontam que, ao decorrer dos anos de 2017 a 2019, houve um aumento na identificação de lesões intraepiteliais escamosas de alto grau, precursora do CCU. Pressupõe-se que tal mudança tenha ocorrido devido a implementação da padronização de critérios citomorfológicos imposta pelo Ministério da Saúde em 2014 para as instituições de análises clínicas vinculadas ao SUS (Magalhães et al. 2020).

Um estudo realizado entre os anos de 2013 a 2017 em dois dos principais laboratórios de citopatologia cervical do Rio de Janeiro, desenvolvido por Rocha et al. 2018, demonstrou bons resultados frente às análises da Unidade Externa de Monitoramento da Qualidade, sendo que dos 19.158 exames investigados apenas 2.481 (12,97\%) foram rejeitados e 441 $(2,65 \%)$ foram considerados insatisfatórios. Segundo os autores, a aplicação do Monitoramento Externo da Qualidade foi essencial para garantir a confiabilidade dos exames citopatológicos e promover a credibilidade dos laboratórios. 
De acordo Branca e Longatto-Filho (2015), o controle de qualidade adequado associado ao treinamento contínuo dos profissionais da área são essenciais para superar as dificuldades e diminuir os resultados falsos-negativos. Uma vez que a leitura e a interpretação das células anormais estão intimamente associadas a execução das etapas anterior do exame e também aos conhecimentos do citologista e seu treinamento básico decorrente da sua formação continuada.

Para Wilson (2015) e Branca e Longatto-Filho (2015), o monitoramento dos padrões de qualidade devem ser prioridade contínua e responsabilidade de todos os profissionais da saúde envolvidos. Além disso, o treinamento de pessoal é de suma importância para manutenção das competências e excelência dos procedimentos em laboratório. Ainda, um fator importante na manutenção do programa de qualidade interno é à escolha do profissional certo para administrar tais atividades, sendo necessário que ele tenha habilidade e comprometimento com o rígido protocolo de controle laboratorial, além de uma personalidade de liderança e boa comunicação interpessoal.

No geral, estudiosos relatam a importância da auditoria e a inspeção do sistema de qualidade nos laboratórios de análise clínica, pois fornecem requisitos para confiabilidade dos serviços oferecidos em laboratório, corroborando com as normas da ISO - International Organization for Standardization, uma agência de referência em serviços de qualidade não governamental responsável por órgãos de padronização nacionais e internacionais em mais de 140 países (Branca e LongattoFilho, 2015). Segundo a ISO 9000, os procedimentos de qualidade garantem a eficácias do processo, sendo assim os laboratórios sob a certificação ISO 9000, 6 devem considerar alguns preceitos da adequação como se diz em ISO 9000:

(1) definir a política de qualidade,

(2) elaborar o organograma do laboratório,

(3) fornecer descrições de funções para todos os membros da equipe,

(4) implementar a política de gestão de recursos humanos, incluindo educação contínua e um sistema de recompensa,

(5) documentar todos os procedimentos, com atenção especial para aqueles relacionados ao Controle de Qualidade, o tratamento das reclamações dos usuários do laboratório e a manutenção dos equipamentos e

(6) definir a organização e sua responsabilidade pela qualidade (ABNT NBR ISO 9000, 2015. p. 10).

Por conseguinte, a implementação de auditorias contribui para continuidade dos padrões de qualidade e favorece o processo contínuo de educação e avaliação crítica de todas as etapas relacionadas aos protocolos de qualidade. No arsenal da Citopatologia, os resultados satisfatórios se relacionam com os cuidados atribuídos aos pacientes e planejamento sistemáticos dos serviços prestados, além de promover uma interação entre os profissionais da saúde e o público-alvo, estabelecer disciplina desde o ato da coleta, no seu manuseamento até o laudo final (Wilson, 2015).

\section{Considerações Finais}

A qualidade do exame Papanicolaou depende do controle de qualidade dos exames citopatológicos uma vez que este fundamenta-se em um agrupamento de medidas empenhadas a detectar, corrigir e diminuir defeito do processo de produtividade dentro do laboratório de Citopatologia, possibilitando assim, o aprimoramento dos procedimentos laboratoriais e minimizando a ocorrência de falhas no diagnóstico, além de contribuir como orientação para o aperfeiçoamento da coleta do material, e instrumento educacional.

A literatura aponta, que para exame de qualidade, o escrutínio deve seguir critérios rigorosos na identificação morfológica das células, com intuito evitar erros e otimizar do desempenho da rotina em laboratório. Outro ponto importante refere-se a coleta, uma vez que muitos dos resultados falsos negativos dos exames Papanicolaou relacionam-se a erros presentes na amostra, cerca de $56 \%$ a $83 \%$, resultando em interpretações errôneas e falsos positivos ou falsos negativos. 
Nota-se que a citopatologia enfrenta dificuldades não apenas na interpretação, mas também durante as diferentes etapas de realização dos exames, que inclui profissionais com diferentes qualificações, experiências e graus de responsabilidades. Dessa forma, o aperfeiçoamento dos profissionais atuantes reflete diretamente na boa qualidade dos exames em geral, sendo enfatizado por inúmeros estudiosos a importância das estratégias voltada para capacitação contínua na prática e teoria da rotina laboratorial.

Portanto, entende-se que na prevenção do CCU, a inovação é um processo contínuo do conhecimento, informação, ações preventivas e trabalho coletivo, cabendo ao profissional biomédico e farmacêutico/bioquímico, como principal protagonista das análises clínicas e competente atuante na citologia dar suas contribuições, fundamentados nos conhecimentos pertinentes inerentes de sua formação, para melhoria e credibilidade dos exames e diagnósticos laboratorial em citopatologia.

\section{Referências}

Amaral, A. F., Araújo, E. S., Magalhães, J. C., Silveira, E. A., Tavares, S. B. N., \& Amaral, R. G. (2014). Impacto da capacitação dos profissionais de saúde sobre o rastreamento do câncer do colo do útero em unidades básicas de saúde. Revista Brasileira de Ginecologia e Obstetrícia, 36, 182-187, FapUNIFESP (SciELO). http://dx.doi.org/10.1590/s0100-7203201400040004.

Agência Nacional de Vigilância Sanitária. (2015). Resolução-RDC nº 302, de 13 de outubro de 2005. Dispõe sobre Regulamento Técnico para funcionamento de Laboratórios Clínicos. Diário Oficial da União, Brasília, DF, 14 out. 2005. Seção 1, p. 33 Apud ABNT NBR ISO 9000: 2015. Sistemas de gestão da qualidade - Fundamentos e vocabulário. ABNT. 2015. https://pncq.org.br/.

Brasil. Ministério da Saúde. (2013). Portaria no 3.388, de 23 de julho de 2013: redefine a qualificação nacional em citopatologia na prevenção do câncer do colo do útero (QualiCito), no âmbito da rede de atenção à saúde das pessoas com doenças crônicas. Diário Oficial da União, 11 p. https://bvsms.saude.gov.br/.

Branca, M., \& Longatto-Filho, A., (2015). Recommendations on Quality Control and Quality Assurance in Cervical Cytology. Acta Cytologica, 59, 361-369, S. Karger AG. http://dx.doi.org/10.1159/000441515.

Clarke, M. A., Fetterman, B., Cheung, L. C., Wentzensen, N., Gage, J. C., Katki, H. A., Befano, B., Demarco, M., Schussler, J., \& Kinney, W. K. (2018). Epidemiologic Evidence That Excess Body Weight Increases Risk of Cervical Cancer by Decreased Detection of Precancer. Journal Of Clinical Oncology, 36, 1184-1191, 20. American Society of Clinical Oncology (ASCO). http://dx.doi.org/10.1200/jco.2017.75.3442.

Galvão, E. F. B., Silva, M. J. M., Esteves, F. A. M., \& Peres, A. L. (2015). Frequência de amostras insatisfatórias dos exames preventivos do câncer de colo uterino na rede pública de saúde, em município do agreste pernambucano: frequency of unsatisfactory samples of preventive exams of cervical cancer in the public health system in a city located in pernambuco, brazil. Revista Paraense de Medicina, 29, 51-56. https://bvsalud.org/.

Instituto Nacional Do Câncer - INCA (Brasil). (2016). Manual de Gestão da Qualidade Laboratório de Citopatologia. (2a ed.), INCA, 160p ISBN 978-857318-281-1. https://www.inca.gov.br/.

International Agency for Cancer Research (IARC). (2019). Estimativa 2020: incidência de câncer. https://gco.iarc.fr/tomorrow/home.

Jakobczynski, J. (2018). Capacitação dos profissionais de saúde e seu impacto no rastreamento de lesões precursoras do câncer de colo uterino. Revista Brasileira de Análises Clínicas, 1, 80-95. 10.21877/2448-3877.201800662

Ministério da Saúde. (2012). Instituto Nacional de Câncer - INCA Manual de gestão da qualidade para laboratórios de Citopatologia. https://www.inca.gov.br/publicacoes/manuais/manual-de-gestao-da-qualidade-para-laboratorio-de-citopatologia.

Machado, E. P., Alves, M. B. M., Irie, M. M. T., Zrzebiela, F. F., Reche, P. M., \& Borato, D. C. K. (2018). Controle interno da qualidade em citopatologia: o dilema da subjetividade. Rev. Brasileira de Análises Clínicas, 50, 244-249. http://dx.doi.org/10.21877/2448-3877.201800662.

Magalhães, J. C. (2020). Avaliação dos indicadores de qualidade dos exames citopatológicos do colo do útero realizados em um município do Paraná, Brasil: evaluation of quality indicators of cervical cytopathology tests carried out in a municipality of paraná, brazil. J Bras Patol Med Lab, 2, 1-7. https://doi.org/10.5935/1676-2444.20200041.

Mori, M. A., \& Ribeiro C. N. M. (2015). Falhas no diagnóstico do câncer de colo uterino. Rev. Eletrônica Biociências, Biotecnologia e Saúde, 2, 37-50. https://interin.utp.br/.

Paula, A. C., Souza, N. G., Prado, T. C., \& Ribeiro, A. A. (2017). Indicadores do monitoramento interno da qualidade dos exames citopatológicos do Laboratório Clínico da Pontifícia Universidade Católica de Goias (PUC-GO). RBAC.49(2):200-5. https://doi.org/10.21877/2448-3877.201700534.

Roever, L. (2017). Compreendendo os estudos de revisão sistemática. Rev Soc Bras Clin Med., 5, 127-130. https://pesquisa.bvsalud.org/.

Rocha, V., Malfacini, S., Gomes, A., \& Rocha, C. (2018). External Quality Monitoring of the Cervical Cytopathological Exams in the Rio de Janeiro City. Revista Brasileira de Ginecologia e Obstetrícia / Rbgo Gynecology And Obstetrics, 40, 338-346, Georg Thieme Verlag KG. http://dx.doi.org/10.1055/s-00381657755 .

Silva, I. C., \& Assis, I. B., (2019). Os efeitos das incorreções pré-analíticas para o exame de papanicolaou. Revista Saúde em Foco, São Lourenço, 11, 876890. https://portal.unisepe.com.br/. 
Research, Society and Development, v. 10, n. 10, e361101019079, 2021

(CC BY 4.0) | ISSN 2525-3409 | DOI: http://dx.doi.org/10.33448/rsd-v10i10.19079

Silva, G. P. F., Cristovam, P. C., \& Vidotti, D. B. (2017). O impacto da fase pré-analítica na qualidade dos esfregaços: cervicovaginais: the impact of the preanalytical phase on the cervical smears quality. Revista Brasileira de Análises Clínicas, 49, 134-139. http://dx.doi.org/10.21877/2448-3877.201600470.

Santos, M. J. S., \& Ribeiro, A. A., (2020). Estratégias Utilizadas para Melhorar a Qualidade dos Exames Citopatológicos. Revista Brasileira de Cancerologia, 66, 1-7. Revista Brasileira De Cancerologia (RBC). http://dx.doi.org/10.32635/2176-9745.rbc.2020v66n1.104.

Ughini, S. F. O. (2016). Importância da qualidade da coleta do exame preventivo para o diagnóstico das neoplasias glandulares endocervicais e endometriais. Revista Brasileira de Analise Clinica, 5, 1-7. http://www.rbac.org.br/.

Wilson, A. (2015). The role of Cytotechnologists in quality assurance and audit in non-gynaecological cytology. Cytopathology, 26, 75-78. Wiley. http://dx.doi.org/10.1111/cyt.12246. 